\title{
A 3D comprehensive finite element based simulation for best Shrink Fit design process
}

\author{
Hossein Golbakhshi $^{1}$, Moslem Namjoo ${ }^{1}$ and Meisam Mohammadi ${ }^{2, a}$ \\ 1 Department of Agricultural Machinery Engineering, Faculty of Agriculture, University of Jiroft, Jiroft, Iran \\ 2 Young Researchers and Elites Club, Kerman Branch, Islamic Azad University, Kerman, Iran
}

Received 2 February 2013, Accepted 11 February 2013

\begin{abstract}
Shrink fits are low-price connections which are widely used in industry and industrial connections. In designing shrink fits it is important to consider radial interface for optimized performance and also to choose an accurate method of fabrication and assembling. Parts which have to be assembled are usually exposed to thermo-mechanical loads. Mode and time duration of heat transfer have a significant effect on required hydraulic force, stress time rate of creation in parts and joint ability to withstand against external loads. Therefore, planning a set of appropriate thermal and structural procedures has significant role in reducing energy consumption, optimized performance and promoting the speed of parts assembly. Despite of the fact, few researches have been done on shrink fit operation and design, rather than dimensional design. In this study, shrink fits are studied in two main processes: first heating and mounting process and then backing to the ambient condition. A 3D coupled thermal and structural simulation based on FEM is done on each process through well-known Solidworks Premium. To evaluate the accuracy, exact analytical solution of two steel rings shrink fit is compared with the approach outcomes. Results of validated method are used for choosing the most optimum sub processes of shrink fit fabrication.
\end{abstract}

Key words: Shrink fit connection / coupled thermal-mechanical analysis / design process / finite element / solidworks

\section{Introduction}

Shrink fits as an interference fitted connections, have been widely used in industrial assemblies due to their simple implementation process, low costs and ability to withstand high loads. Because of differences between the internal diameter of the hub and an external diameter of the shaft, which is called the radial interference, a contact pressure is created on the contacting surface of two bodies. In applications such as turbine disks, rotors for electric motors, mounting the gear and pulleys on the shafts or locating ball and roller bearing, the friction between the pressed surfaces causes a connection tolerance against twisting and axial loads.

However the dimensions and mechanical properties are not the only factors that affect the gradients of displacement and stress in the jointed bodies. In shrink fit assemblies with considerable radial interference, before locating the parts, heat treatment is used so that the external parts are heated or the shaft is cooled and then they are allowed to return back the operating temperature.

\footnotetext{
a Corresponding author:

meisam.mohammadi@hotmail.com
}

Therefore, stresses are generated during the process of mounting especially in shrink fits which have two main sources, penetration of two bodies and thermal strains in cooling stage. It is clear that increasing the radial interference increases the amount of stresses in both shaft and hub. It is important to note that the effective stresses generated in shrink fit during the mounting process together with the internal effects arising from the applied loads must not exceed strength of the parts. On the other hand, if the contact pressure between neighboring surfaces is not enough, relative circumferential slip may occur which depending on the magnitude of the slip, both fretting wear and fatigue may be potential causes of premature failure [1]. For these reasons, it is essential to have an accurate design for dimensions of shrink fit.

However, thermal strains that strongly depend on intensity and time duration of cooling stage have a great effect on the joint strength and displacement field of the bodies. In addition, economical consideration plays a major role in optimization of related industrial processes. So, shrink fit can be regarded as a set of thermo-mechanical sub-process where using numerical analysis of each step, the most appropriate method can be selected. 


\section{Nomenclature}

\begin{tabular}{|ll|}
\hline$A$ & Contacting surface area $\left(\mathrm{m}^{2}\right)$ \\
$C$ & Specific heat $\left(\mathrm{J} \cdot \mathrm{kg}^{-1} \cdot \mathrm{K}^{-1}\right)$ \\
$E$ & Young modulus $(\mathrm{Pa})$ \\
$F$ & Force $(\mathrm{N})$ \\
$h$ & Thermal contact conductance $\left(\mathrm{J}^{-} \mathrm{m}^{-2} \cdot \mathrm{K}^{-1}\right)$ \\
$k$ & Thermal conductivity $\left(\mathrm{W} \cdot \mathrm{m}^{-1} \cdot \mathrm{K}^{-1}\right)$ \\
$l$ & Length of ring $(\mathrm{m})$ \\
$N u$ & Nusselt number $(-)$ \\
$p$ & Pressure $(\mathrm{Pa})$ \\
$p r$ & Prandtl number $(-)$ \\
$r, \theta, z$ & Cylindrical coordinates $(\mathrm{m}, \mathrm{rad}, \mathrm{m})$ \\
$R e$ & Reynolds number $(-)$ \\
$u$ & Displacement component in radial direction $(\mathrm{m})$ \\
$\alpha$ & Thermal expansion coefficient $\left(\mathrm{K}^{-1}\right)$ \\
$\delta$ & Radius variation $(\mathrm{m})$ \\
$\varepsilon$ & Strain $(-)$ \\
$\mu$ & Coefficient of friction $(-)$ \\
$\rho$ & Mass density (kg.m $\left.{ }^{-3}\right)$ \\
$\sigma$ & Stress (Pa) \\
$v$ & Poisson ratio $(-)$ \\
\hline & $\quad$ Subscripts \\
\hline $\mathrm{c}$ & Contact \\
$\mathrm{i}$ & Inner \\
$\mathrm{o}$ & Outer \\
\hline
\end{tabular}

In scientific studies, the highest priority is to find analytical solution for the problems. Because of complex geometry and difficulties in analysis, there are a little theoretical study in the literature (Pedersen [2] and Antoni and Gaisne [3]) the stress analysis of shrink fit process is performed through. The Lame's equations, which are available in classical literature of mechanics [4-6]. Finite extent of the contact surface area, local variations in thermal gradient in the neighboring of parts and friction between the contact layers will lead to not accurate estimation of process by these equations [7]. It is worth to mention that these equations ignore the effect of thermal processes and have no idea about the change rate of stresses and strains. However, because of its popularity and acceptability, the obtained results from Lame's equations have been used as a criterion for assessing the validity of the other developed numerical methods.

Today, many practical engineering problems are investigated through several FEM based commercial software(s) to consider real geometry and working conditions. The analysis done by Parasad et al. [8], can be cited as an appropriate study among those carried out on shrink fit. In this study, stress distribution of a hollow shaft and hub system is presented for various values of length to diameter and outer diameter to inner diameter ratios. Using finite element method, Ozel et al. [9] analyzed the stress and deformations in shrink fitted joints for various fit forms. Mack and Bengri [10] done the solution of a transient elastic-plastic thermal stress problem for a shrink fit with solid inclusion. In this regard, with including the effects of thermal strains, Zhang [11] analyzed the stress generated at interface of some shrink fitted connections such as ring gear and wheel systems.
In the present study, shrink fitted joints are investigated theoretically to improve the designing process of assembling parts in the industrial assemblies. Therefore, to determine the stresses and deformations in the bodies, a concurrent thermal and structural analysis of two metal rings with specific dimensions and mechanical properties is performed by including the mode of heat transfer and change in the contact resistance resulted from increasing thermal strains. Comparing the obtained results with those of theoretical results in the literature shows the accuracy of the method.

\section{Structural analyses}

When two cylindrical parts are connected by shrink fitting, a contact pressure is created between the contacted bodies. In order to determine the resultant stresses from this contact pressure, a model of thick walled vessel subjected to pressure is used. Ignoring the body force components, the general equations of equilibrium in cylindrical coordinates are

$$
\begin{aligned}
\frac{1}{r} \frac{\partial}{\partial r}\left(r \sigma_{r r}\right)+\frac{1}{r} \frac{\partial}{\partial \theta}\left(\sigma_{r \theta}\right)+\frac{\partial}{\partial z}\left(\sigma_{r z}\right)-\frac{\sigma_{\theta \theta}}{r} & =0 \\
\frac{1}{r^{2}} \frac{\partial}{\partial r}\left(r^{2} \sigma_{r \theta}\right)+\frac{1}{r} \frac{\partial}{\partial \theta}\left(\sigma_{\theta \theta}\right)+\frac{\partial}{\partial z}\left(\sigma_{z \theta}\right) & =0 \\
\frac{1}{r} \frac{\partial}{\partial r}\left(r \sigma_{z r}\right)+\frac{1}{r} \frac{\partial}{\partial \theta}\left(\sigma_{z \theta}\right)+\frac{\partial}{\partial z}\left(\sigma_{z z}\right) & =0
\end{aligned}
$$

Since the thick walled cylinder is assumed to be axisymmetric, therefore the stress components are not function of $\theta$ and also $\sigma_{r \theta}=0$ due to the symmetry. Also, it is assumed that the longitudinal elongation is constant on the cross section and any right section of the cylinder remains plane after stressing. So the above three equilibrium equations can be reduced to the following one

$$
\frac{\mathrm{d}}{\mathrm{d} r}\left(\sigma_{r r}\right)+\frac{\sigma_{r r}-\sigma_{\theta \theta}}{r}=0
$$

Also, the strain displacement equations for axi-symmetric problems are

$$
\begin{aligned}
& \varepsilon_{r r}=\frac{\mathrm{d} u_{r}}{\mathrm{~d} r} \\
& \varepsilon_{\theta \theta}=\frac{u_{r}}{r}
\end{aligned}
$$

where $u_{r}$ is the displacement field in the radial direction. Using Hook's law and the above equations, following differential equation is obtained for determining the function $u_{r}$

$$
\frac{\mathrm{d}^{2} u_{r}}{\mathrm{~d} r^{2}}+\frac{1}{r} \frac{\mathrm{d} u_{r}}{\mathrm{~d} r}-\frac{u_{r}}{r^{2}}=0
$$

Therefore, it is easy to show that stress components are obtained as

$$
\begin{aligned}
& \sigma_{r r}=\frac{1}{r_{\mathrm{o}}^{2}-r_{\mathrm{i}}^{2}}\left(\left(p_{\mathrm{i}} r_{\mathrm{i}}^{2}-p_{\mathrm{o}} r_{\mathrm{o}}^{2}\right)+\left(\frac{r_{\mathrm{i}}^{2} r_{\mathrm{o}}^{2}\left(p_{\mathrm{o}}-p_{\mathrm{i}}\right)}{r^{2}}\right)\right) \\
& \sigma_{\theta \theta}=\frac{1}{r_{\mathrm{o}}^{2}-r_{\mathrm{i}}^{2}}\left(\left(p_{\mathrm{i}} r_{\mathrm{i}}^{2}-p_{\mathrm{o}} r_{\mathrm{o}}^{2}\right)-\left(\frac{r_{\mathrm{i}}^{2} r_{\mathrm{o}}^{2}\left(p_{\mathrm{o}}-p_{\mathrm{i}}\right)}{r^{2}}\right)\right)
\end{aligned}
$$


In the above relations subscripts $\mathrm{i}$ and o represent the internal and external parameters, radius and pressure, respectively. Equations (9) and (10) are known as the Lame's equations.

Since in a shrink fit assembly of two cylinders, the inner member undergoes external pressure and internal pressure is exerted to the outer member, equations (8) and (9) can be easily used for determination of radial and tangential stresses at any point in either cylinder. The contact pressure needed in the above relations is obtained through the total deformation. Hence

$$
\delta=\delta_{\mathrm{o}}-\delta_{\mathrm{i}}
$$

where $\delta_{\mathrm{i}}$ and $\delta_{\mathrm{o}}$ are variations in radius of inner and outer member, respectively. Then

$$
p=\frac{\frac{\delta}{r_{2}}}{\frac{1}{E_{\mathrm{o}}}\left(\frac{r_{3}^{2}+r_{2}^{2}}{r_{3}^{2}-r_{2}^{2}}+v_{\mathrm{o}}\right)+\frac{1}{E_{\mathrm{i}}}\left(\frac{r_{2}^{2}+r_{1}^{2}}{r_{2}^{2}-r_{1}^{2}}-v_{\mathrm{i}}\right)}
$$

In equation (11), $E$ and $v$ are Young modulus and Poisson ratio, respectively. Also, $r_{1}, r_{2}$ and $r_{3}$ are internal radius of inner ring, external radius of inner ring or internal radius of outer ring and external radius of outer ring, respectively. Furthermore, knowing the amount of contact pressure $p$, radial displacement at any point within the domain of two bodies can be obtained:

$$
\begin{aligned}
& u_{\text {inner }}=\frac{1-v_{\mathrm{i}}}{E_{\mathrm{i}}}\left(-\frac{r_{2}^{2} p}{r_{2}^{2}-r_{1}^{2}}\right) r+\frac{1-v_{\mathrm{i}}}{E_{\mathrm{i}}}\left(-\frac{r_{1}^{2} r_{2}^{2} p}{r_{2}^{2}-r_{1}^{2}}\right)\left(\frac{1}{r}\right) \\
& u_{\text {outer }}=\frac{1-v_{\mathrm{o}}}{E_{\mathrm{o}}}\left(\frac{r_{2}^{2} p}{r_{3}^{2}-r_{2}^{2}}\right) r+\frac{1-v_{\mathrm{o}}}{E_{\mathrm{o}}}\left(\frac{r_{2}^{2} r_{3}^{2} p}{r_{3}^{2}-r_{2}^{2}}\right)\left(\frac{1}{r}\right)
\end{aligned}
$$

In a special case that the two members are made of the same material, equation (11) can be simplified as

$$
p=\frac{E \delta}{r_{2}}\left(\frac{\left(r_{3}^{2}-r_{2}^{2}\right)\left(r_{2}^{2}-r_{1}^{2}\right)}{2 r_{2}^{2}\left(r_{3}^{2}-r_{1}^{2}\right)}\right)
$$

\section{Thermal analysis}

Heat treatment is a significant step in shrink fitting, especially in heavy fits. The proper interference is done as expanding the larger part by heating and contraction of smaller part by cooling. Thus, the inclusion of thermal effects in the analysis is inevitable. The most important part in thermal analysis is the determination of the convective coefficient of heat transfer between fluid flow and external surfaces of the two cylinders.

Morgan has presented a comprehensive overview of the relations obtained for convection between a cylinder and fluid flow [12]. Among them, Churchill and Bernstein

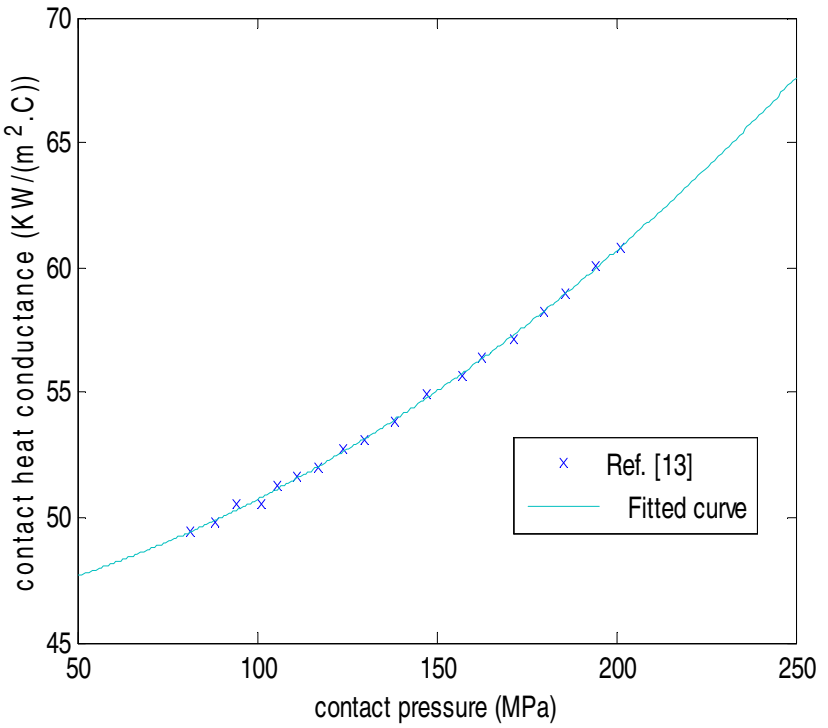

Fig. 1. Contact conductance versus pressure for steel surfaces in air.

equation in the wide range of Reynolds and Prandtl numbers, gives the average Nusselt number on the cylinder surface as

$$
\overline{N u_{D}}=0.3+\frac{0.62 \sqrt{R e_{\mathrm{D}}} \sqrt[3]{\mathrm{Pr}}}{\left(1+\left(\frac{0.4}{\mathrm{Pr}}\right)^{\frac{2}{3}}\right)^{\frac{1}{4}}}\left(1+\left(\frac{R e_{\mathrm{D}}}{28200}\right)^{\frac{5}{8}}\right)^{\frac{4}{5}}
$$

which its results with a good accuracy, describes the heat transfer between the cylindrical surfaces and the fluid flow.

During the cooling phase, as the magnitude of contact pressure increases, surface roughness in the neighboring regions, that is considered as the main source of thermal contact resistance, will sharply reduce. So, the variation of thermal resistance with respect to the pressure should be known for the given materials.

The changes in thermal conductance are determined from reference [13], for a wide range of contact pressures. Figure 1 shows the trends in terms of metric units. As depicted, quadratic function $h_{\mathrm{c}}=0.00026 p^{2}+0.022 p+$ 46 will conveniently reproduce the available experimental results. It seems reasonable to consider a constant contact resistance in each enough small interval for the analysis. Knowing the thermal conductance, the resistance between two bodies can be easily determined for given contact pressure as

$$
R_{\text {contact }}=\frac{1}{h_{\mathrm{c}} A}
$$

where $A$ is the contact surface area between two bodies and $h_{\mathrm{c}}$ is the thermal conductance in the neighboring surface.

\section{FEM analysis}

As it was reviewed before, most of the studies were about design and performance estimation of fitted joints 


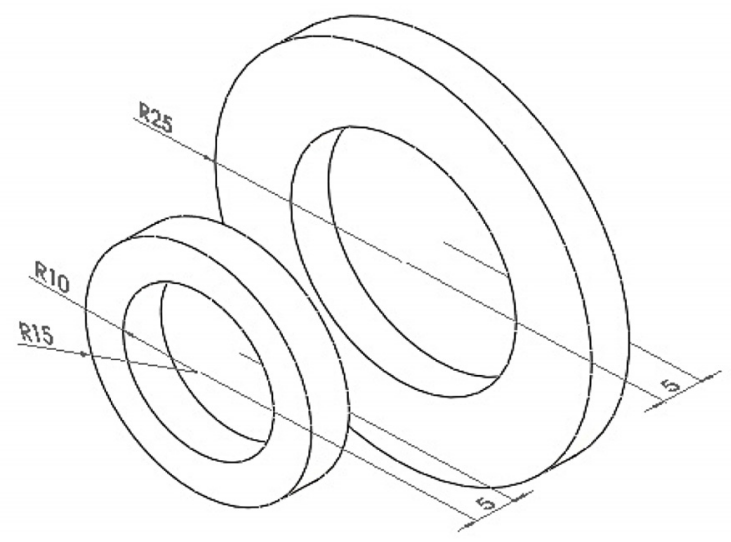

(a)

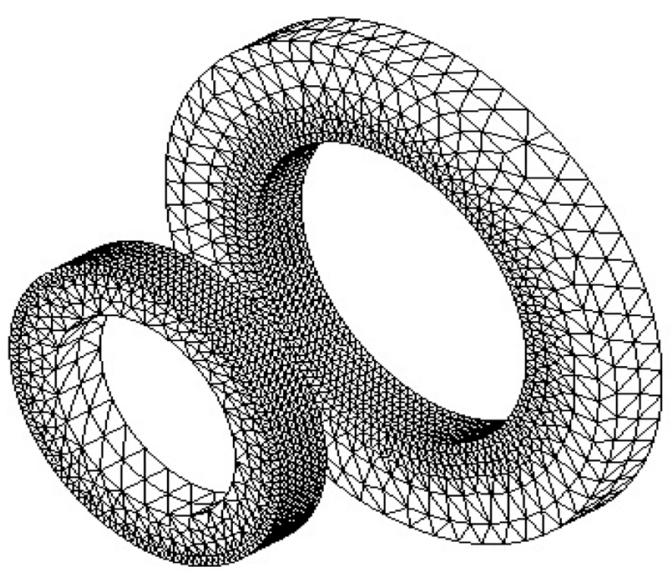

(b)

Fig. 2. Rings dimensions (a) and applied mesh for modeling (b).

Table 1. Mechanical properties of rings.

\begin{tabular}{cc}
\hline Property & Value \\
\hline Elastic modulus & $210(\mathrm{GPa})$ \\
Poisson's ratio & 0.28 \\
Mass density & $7700 \mathrm{~kg} \cdot \mathrm{m}^{-3}$ \\
Yield strength & $620(\mathrm{MPa})$ \\
Ultimate strength & $724(\mathrm{MPa})$ \\
Thermal expansion coefficient & $1.3 \times 10^{-5} \mathrm{~K}^{-1}$ \\
Thermal conductivity & $50 \mathrm{~W} \cdot \mathrm{m}^{-1} \cdot \mathrm{K}^{-1}$ \\
Specific heat & $460 \mathrm{~J} \cdot \mathrm{kg}^{-1} \cdot \mathrm{K}^{-1}$ \\
\hline
\end{tabular}

under different loading conditions and the fabrication process of such connections was not considered so much. Whereas the time duration and mode of heat transfer for heating and cooling plays a significant role in stress and strain rate creation in the parts. In this study, numerical analysis of shrink fit is done for two steps, the outer body is heated and cooled to the environmental conditions.

In the following a shrink fitting of two steel rings with specified dimensions as shown in Figure 2 is done based on the prescribed approach. The mechanical properties of steel are tabulated in Table 1.

The first step is just a heat transfer analysis between a cylindrical body and environment with flame temperature of $1000{ }^{\circ} \mathrm{C}$ and convection coefficient of $50 \mathrm{~W} \cdot \mathrm{m}^{-2} \cdot \mathrm{K}^{-1}$. In this step, there is no stress associated with the thermal strains produced via heat transfer. So, the first part of thermal analysis is confined to determination of the temperature gradient and thermal strains of the outer body due to exerted heat flux on some parts of its external surfaces. It is assumed that yield strength is independent of temperature variation and hence has no effect on the accuracy of the results. The other step is a FEM coupled thermal-structural simulation by considering free convection with a coefficient of $5 \mathrm{~W} \cdot \mathrm{m}^{-2} \cdot \mathrm{K}^{-1}$ and radiation with emissivity of 0.3 from the heated parts to the ambient.
As shown in Figure 2, totally 11709 curved based solid elements with 4 Jacobian points are used for discretization of problem domain and evaluation of arising integrals. The "node to surface" contact condition is defined on the neighborhood region, and due to intense stress gradient, a finer mesh is used around this region.

At each time interval of the investigation in which the thermal contact resistance is considered to be constant, the results of thermal study on temperature and displacement gradients is a prerequisite stage for structural analysis. After determination of contact pressure, one can estimate the thermal conductance on shrink fit region for thermal study of next time interval of the analysis. Thus for each point in the problem domain, desired outputs can be accessed over time.

Finally, displacements and stresses are calculated for specified points within the domain of the problem. Comparing the results with theoretical solutions indicates the high accuracy of the proposed approach.

\section{Results and discussions}

For modeling and analysis, a finite element method (FEM) based software, Sloidworks, is used. In Figure 3, the temperature gradient of the heated outer ring after $30 \mathrm{~s}$ is shown. Due to size expansion of the outer ring resulted from the thermal strains, the force required for fitting the inner ring which is obtained from the following relationship dramatically decreases.

$$
F=2 \mu l \pi r_{\mathrm{c}} p_{\mathrm{c}}
$$

in which $r_{\mathrm{c}}$ and $p_{\mathrm{c}}$ are radius and pressure in the contact region, respectively and $\mu$ is the coefficient of friction between the two rings which is assumed to be 0.05 according to their contacting material. Figure $3 \mathrm{~b}$ illustrates the variation of contact pressure with respect to the heating time. 


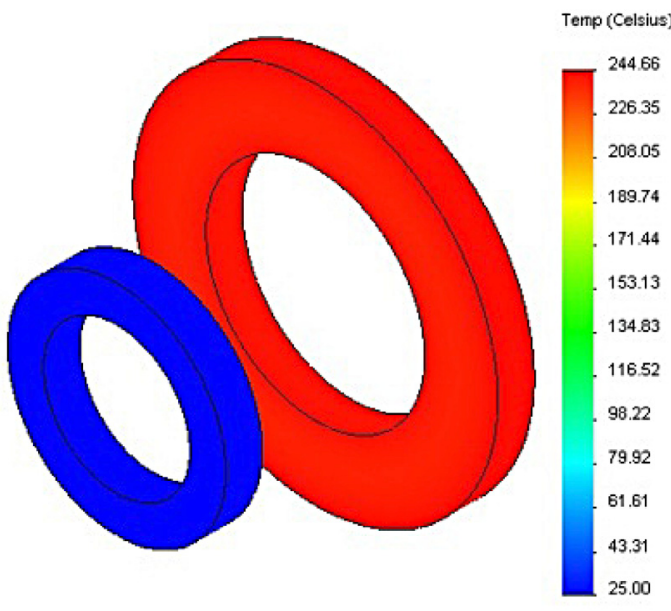

(a)

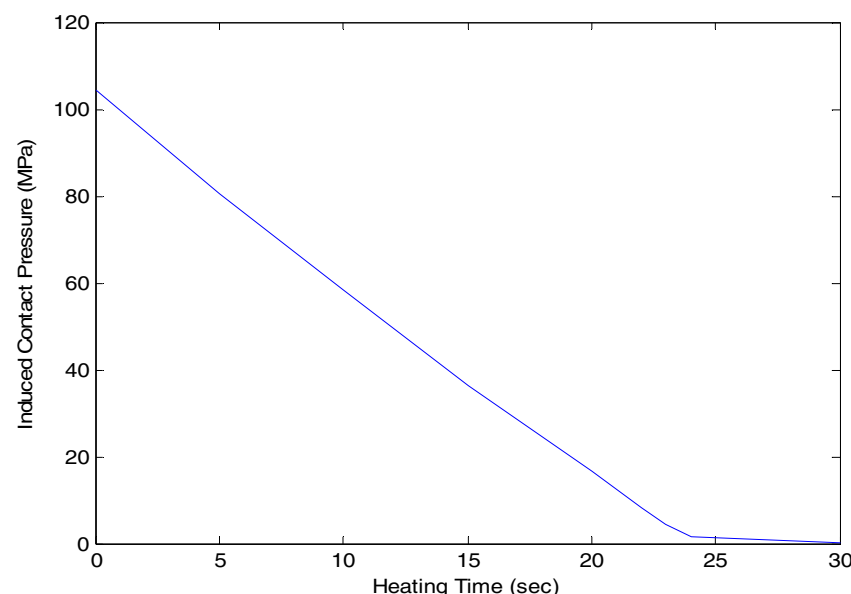

(b)

Fig. 3. Results for outer ring warming. (a) Temperature gradient after $30 \mathrm{~s}$. (b) Contact pressure resulted from two rings insertion heating period.

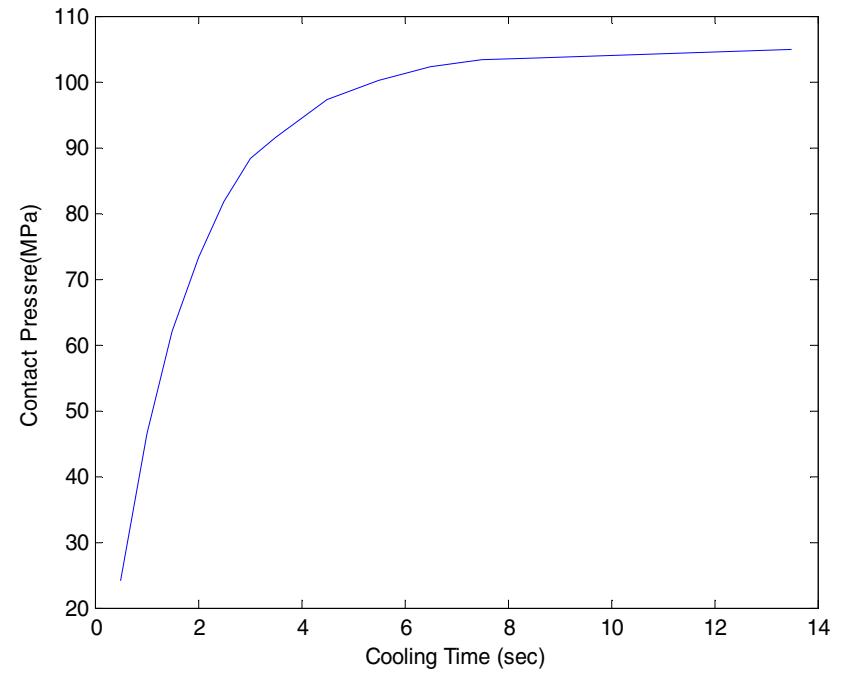

Fig. 4. The contact pressure variation versus the cooling stage.

It is obvious that less amount of contact pressure and frictional resisting force leads to faster and easier insertion in shrink fit process. However it can be found from the diagram that after about $24 \mathrm{~s}$ the contact pressure reaches to its minimum value and any more heating is just wasting the cost and energy.

Just after mating the two rings, thermal conduction between the bodies and radiation/convection heat exchange with surrounding occurs because of temperature difference. As stated before, the thermal conductance is highly dependent on the neighboring region of contact pressure. So by adequate choice of increments, the thermal resistance will not drop more than $2 \%$ and can be considered constant in each stage of the analysis.
Variation of contact pressure versus cooling time is plotted in Figure 4. It is clear that although the cooling time is increased, the contact pressure remains constant.

In Figure 5a, a comparison between the presented result and theoretical results is done which show a good accuracy in the results. Also, in Figures 5b and c tangential and radial stresses are plotted for the inner and outer rings in different times.

By determination of thermal contact resistance, thermal solver provides needed data for structural solver to simulate the rings penetration. Therefore, thermal conductance for the next step and also stress/strain gradients are determined. Figure 6 shows the results obtained for stress gradients at three different time steps of shrink fit cooling process. In addition, the displacement of some selected points on the problem domain is depicted in Figure 7.

Figure 8a, shows the analysis of the cooling phase for different convective coefficients. It is clear that changes in heat transfer coefficient have a negligible effect on the results. Also, in Figure 8b variation of contact pressure versus the cooling time for two cases of time duration is plotted. As figure represents, if two rings inserted $20 \mathrm{~s}$ after starting of heating phase, the time rate of increase in generated stresses will change.

\section{Conclusion}

In this paper, thermo-mechanical connection of two steel rings is elaborately analyzed using two thermal and structural solvers and taking into account the convective heat transfer with variable thermal contact resistance. The aim was to determine optimum conditions for better fabrication and design of the shrink fitting of two rings. Comparing of the steady state radial stresses derived from the last time step of the analysis with the theoretical results demonstrates the method's accuracy. 


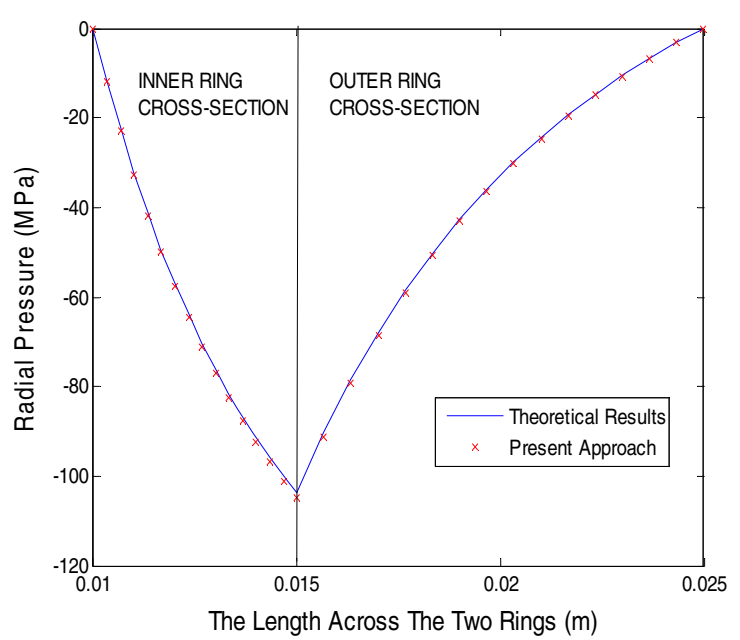

(a)

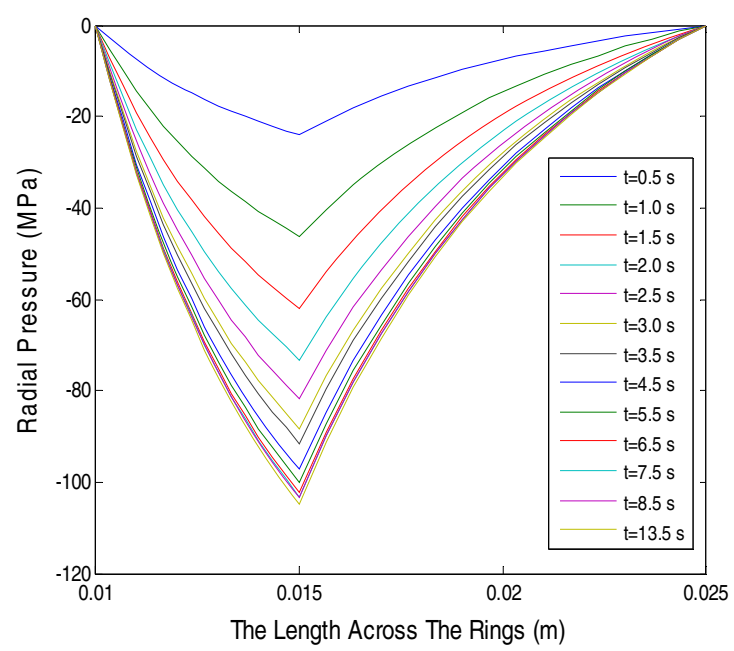

(b)

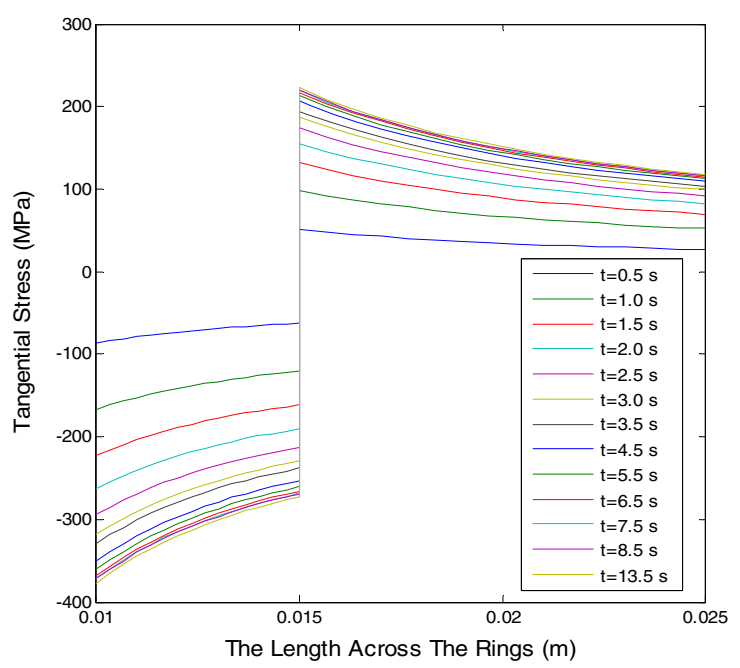

(c)

Fig. 5. Results for the inner and outer rings. (a) Comparison of the presented results with the theoretical results. (b) Variation of radial pressure vs. length across the rings. (c) Variation of tangential pressure vs. length across the rings.

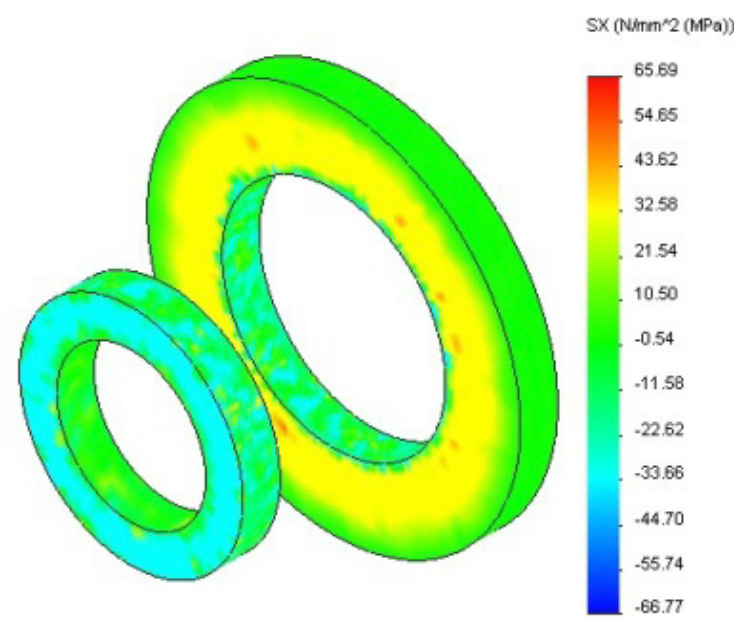

(a)

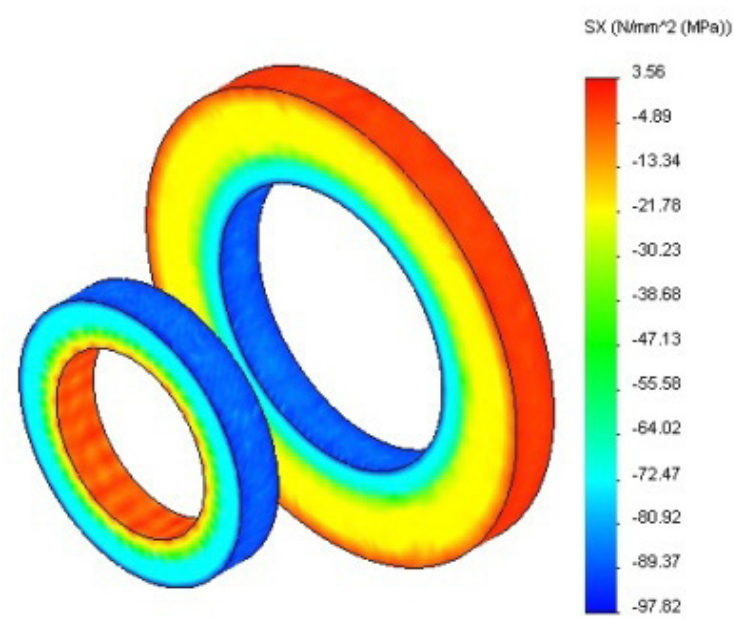

(b)

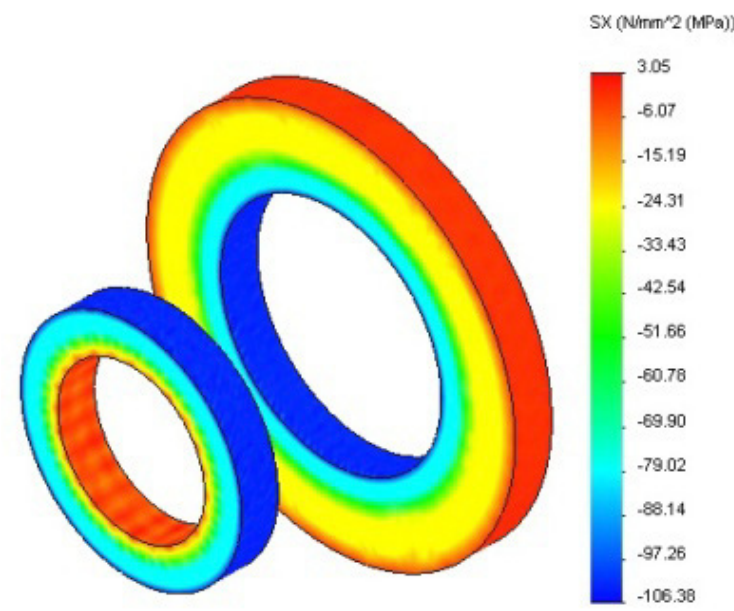

(c)

Fig. 6. Distribution of stress gradient for three different time steps. (a) First time step (0.5 s). (b) Second time step (3.5 s). (c) Third time step $(13.5 \mathrm{~s})$. 


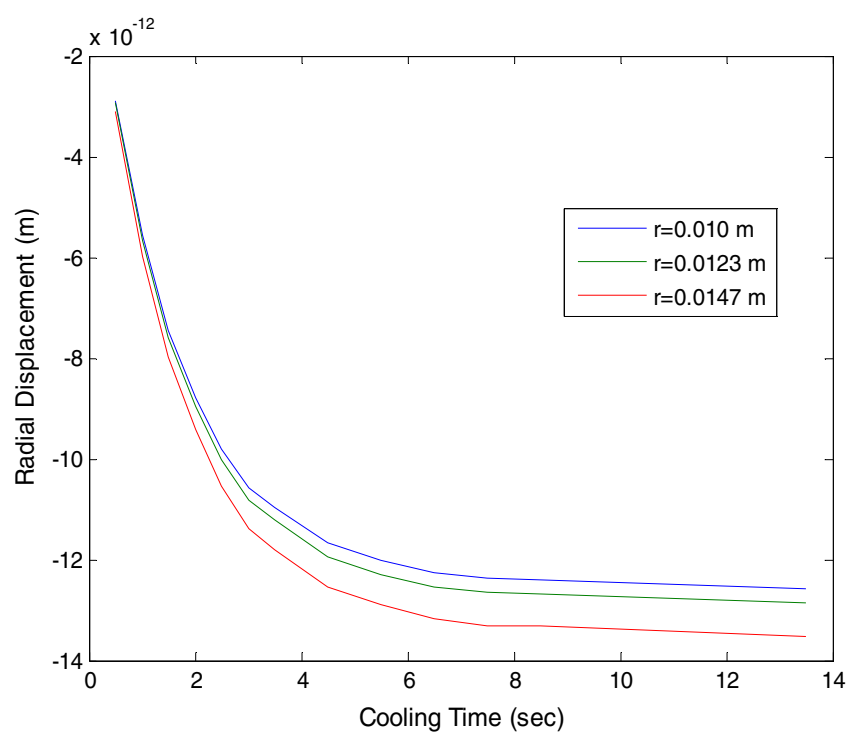

(a)

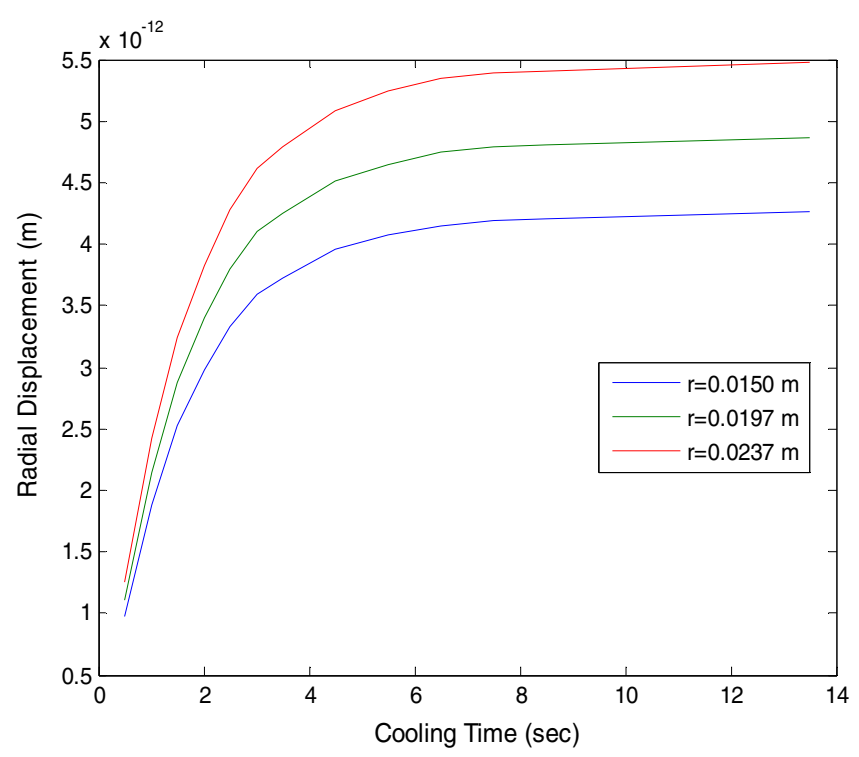

(b)

Fig. 7. Displacement of some selected points during the cooling time. (a) Inner ring. (b) Outer ring.

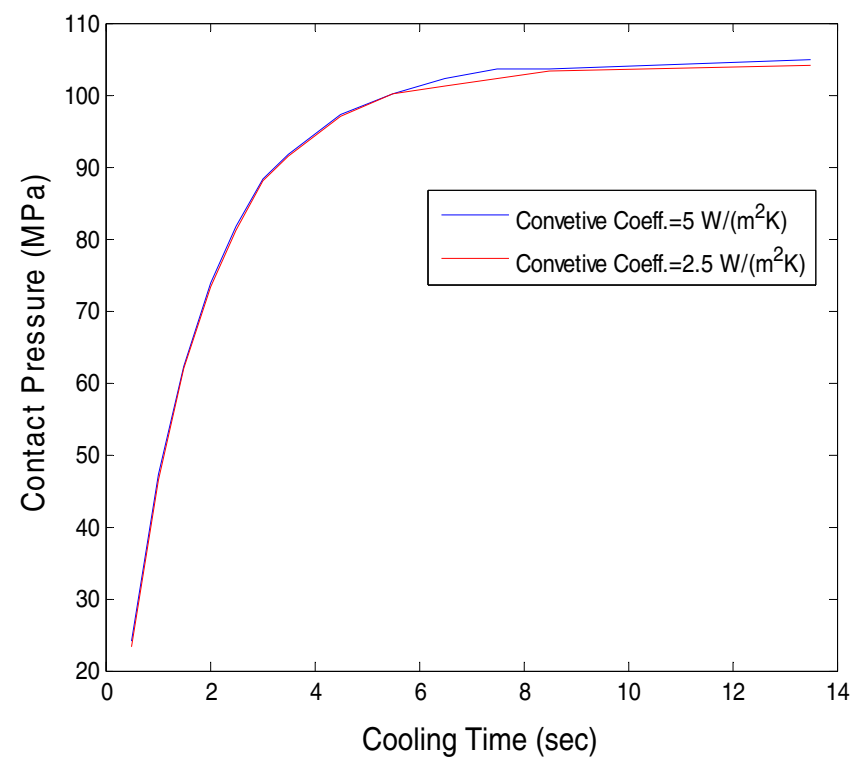

(a)

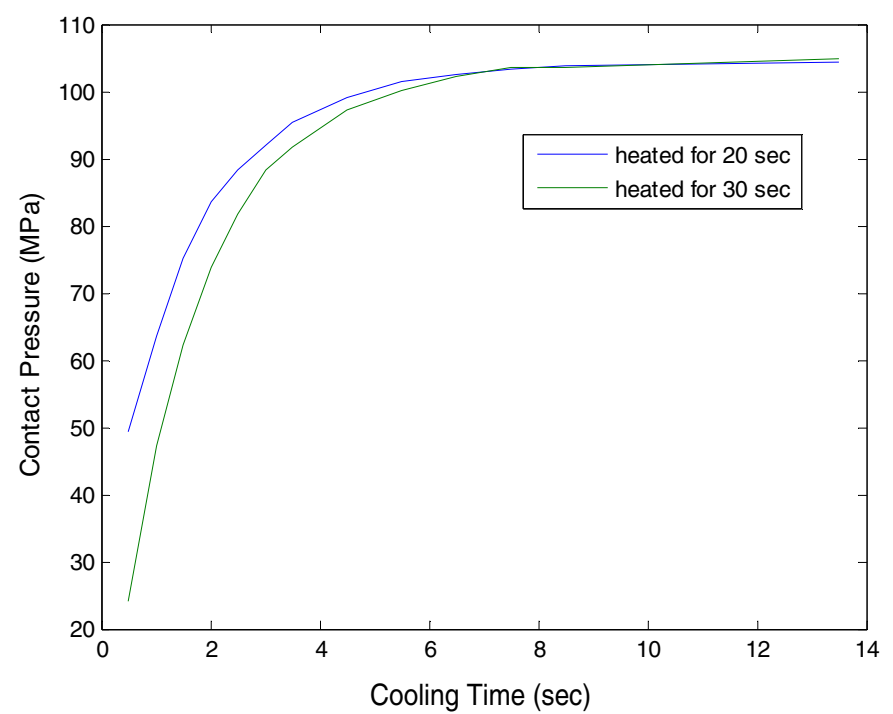

(b)

Fig. 8. The effect of variation of heat transfer mode on contact pressure. (a) Different conductive coefficients. (b) Different heating times.

It was shown that during the heating stage, there is a certain limit in expansion of the outer ring in which the radial interference reaches a minimum value and more heating is just energy wasting. By choosing an appropriate time for this process, besides saving time and money, one can prevent the occurrence of extreme temperature gradients with destructive effect on the outer ring strength.

It is inferred from the results that in the early stages of cooling, the contact pressure between the parts greatly increases which cause to generate the stresses and also displacement gradients are created in the rings. The increase in amount of generated stresses with such a high rate leads to local plastic zones with residual stresses that may affect the strength of loaded joint. Although the insertion of less heated rings produces greater stresses at early stages of cooling phase, but the change rate intensity of contact stress diminishes. Clearly, the longer time the contact stress reaches its final value, gives sufficient time to the strains to develop and therefore the shrink fit would be more tolerable. Thus, by choosing an adequate time for heating, the required force for insertion and the 
stress change rates can be operationally optimized during the fabrication.

\section{References}

[1] C.E. Truman, J.D. Booker, Analysis of a Shrink Fit Failure on a Gear Hub/Shaft Assembly, Eng. Fail. Anal. 14 (2007) 557-572

[2] P. Pedersen, On shrink fit analysis and design, Comput. Mech. 37 (2006) 121-130

[3] N. Antoni, F. Gaisne, Analytical Modeling for Static Stress Analysis of Pin-Loaded Lugs with Bush-Fitting, Appl. Math. Model. 35 (2011) 1-21

[4] K.J. Gohnson, Contact Mechanics, Cambridge University Press, Cambridge, UK, 1985

[5] J.E. Shigley, C.R. Mischke, Standard Handbook of Machine Design, McGraw-Hill, New York, 1985

[6] A.C. Ugural, S.K. Fenseter, Advanced Strength and Applied elasticity, PTR Prentice- Hall, Englewood Cliffs (NJ), 1987
[7] S. Sen, B. Aksakal, Stress Analysis of Interference Fitted Shaft-Hub System under Transient Heat Transfer Conditions, Mater. Des. 25 (2005) 407-417

[8] N.S. Parasad, P. Sashikant, V. Ramamurt, Stress Distribution in Interference Joints, J. Compos. Struct. 51 (1994) 535-540

[9] A. Ozel, S. Temiz, M.D. Aydin, S. Sen, Stress Analysis of Shrink-fitted Joints for Various Fit Forms via Finite Element Method, Mater. Des. 26 (2005) 281-290

[10] W. Mack, M. Bengri, Thermal Assembly of an ElasticPlastic Shrink Fit with Solid Inclusion, Int. J. Mech. Sci. 36 (1994) 699-705

[11] Y. Zhang, B. McClain, X.D. Fang, Design of interference fits via finite element, Int. J. Mech. Sci. 42 (2000) $1835-1850$

[12] V.T. Morgan, The overall convective heat transfer from smooth circular cylinders, in: T.F. Irvine, Jr, J.P. Hartnett (eds.), Adv. Heat Transf. 11 (1975) 199-264

[13] B.B. Mikic, W.M. Rohsenow, Thermal contact resistance, Department of Mechanical Engineering, Massachusetts Institute of Technology, 1966 